\section{Моделирование движения лесовозных автопоездов с использованием ПЭВМ}

\author{
И. Р. Шегельман ${ }^{1}$ \\ Петрозаводский государственный университет \\ В. И. Скрыпник \\ КарНИИЛПК ПетрГУ \\ А. В. Пладов \\ ОАО «Шуялес»
}

\begin{abstract}
АННОТАЦИЯ
В статье дано описание алгоритма и программы расчета показателей движения лесовозных автопоездов на основе новых методов тяговых расчетов, обеспечивающих в сравнении с традиционными методами более высокую точность и достоверность. Алгоритмом программы предусмотрен расчет показателей движения во всех режимах (работа двигателя на внешней и частичных характеристиках, движение накатом, торможение двигателем, моторным тормозом, колесным тормозом) с учетом ограничений скорости. Приведены методика и результаты расчетноэкспериментальных исследований. Обозначена область применения, приведены результаты работы.
\end{abstract}

Ключевые слова: лесовозные автопоезда, алгоритм, программа моделирования движения, производительность автопоезда, экспериментальные исследования, технико-экономические показатели.

\section{SUMMARY}

In the work the description of algorithm and program of calculation of parameters of movement haul rig truckand-trailer is given on the basis of new methods of hauling capacity calculation providing in comparison with traditional methods higher accuracy and reliability. The algorithm of the program stipulates determination of parameters of movement in all modes (work of the engine on external and partial characteristics, movement outwards, braking by the engine, motor brake, wheel brake) taking into account the restrictions of speed. Some results of solving-experimental researches are given. The area of application is designated, the results of work are given.

Keywords: haul rig truck-and-trailer, algorithm, program of modeling of movement, productivity of the haul rig truck-and-trailer, experimental researches, technical and economic parameters.

Вопросы эффективной эксплуатации лесовозного автотранспорта, качества проектирования и строительства лесовозных дорог приобретают все большее значение. Это связано с тем, что наряду с вывозкой

\footnotetext{
${ }^{1}$ Авторы - соответственно зав. каф. ТОЛК, зав. сектором КарНИИЛПК, ген. директор ОАО «Шуялес»

(C) И. Р. Шегельман, В. И. Скрыпник, А. В. Пладов, 2003
}

леса на нижние склады потребителей в хлыстах возрастают объемы вывозки леса автопоездами в сортиментах с лесосек или промежуточных складов непосредственно потребителям и на перерабатывающие предприятия, что приводит к увеличению расстояния доставки леса транспортом лесозаготовительных предприятий и затрат на транспортировку в структуре затрат на заготовку.

В настоящее время автомобильными заводами России, Белоруссии и Украины выпускаются автопоезда для транспортировки хлыстов и сортиментов. Широко применяются при транспортировке сортиментов зарубежные автопоезда-сортиментовозы фирм Сису, Скания и др.

В связи с разнообразием типов автопоездов и условий их эксплуатации весьма актуальны многовариантные задачи выбора оптимального типа автопоезда для конкретной автодороги, схемы вывозки (одноступенчатая, двухступенчатая), определение производительности и технико-экономических показателей работы лесовозных автопоездов.

Как правило, эти задачи решаются либо с использованием укрупненных нормативов, которые разработаны для усредненных условий эксплуатации без учета особенностей плана и профиля конкретной автодороги, либо с использованием традиционных методов тяговых расчетов, которые крайне упрощены и не дают возможности эффективно решать заданные задачи ввиду недостаточного учета влияющих факторов, малой точности и достоверности.

Решение указанных и других технико-экономических задач, возникающих в процессе эксплуатации лесотранспорта, возможно лишь при условии достаточно точного определения скорости, времени движения автопоездов, расхода топлива с учетом всех факторов, влияющих на показатели движения.

Ранее $[1,2]$ выведены зависимости для определения скорости, времени движения, расхода топлива лесовозных автопоездов во всех возможных режимах движения, с учетом основных факторов, воздействующих на движение автопоездов (типа и состояния покрытия, особенностей плана и профиля, непрерывного изменения уклона на вертикальных кривых, дополнительного сопротивления на горизонтальных кривых, сопротивления воздушной среды, изменения основного сопротивления с изменением скорости, динамических качеств автопоездов). Для использования в расчетных формулах выведены эмпирические зависимости, аппроксимирующие кривые тяговой и тормозной характеристик автомобилей, и зависимости расхода топлива от числа оборотов скоростной характеристики двигателя [3].

Алгоритмом программы моделируется движение лесовозных автопоездов с учетом ограничений скорости; рассчитывается скорость движения при разгоне и замедлении в тяговом и тормозном режиме (торможение двигателем, моторным тормозом, ко- 
лесными тормозами), при движении накатом, моделируется переключение передач, снижение скорости к участкам ограничений, движение на участках ограничений в различных режимах; определяется расход топлива.

Ранее были разработаны табличные и номографические методы расчета скорости и времени движения лесовозных автопоездов с использованием выведенных зависимостей и разработана программа расчета показателей движения применительно к ПЭВМ «Минск-32» и ЕС-1022, которые использовались в системах автоматизированного проектирования лесовозных автодорог САПАД-1 и САПАД-ЕС для оценки качества проектируемых дорог с учетом транспортной составляющей и оптимизации положения проектной линии продольного профиля [5,6]. Однако ввиду отсутствия ПЭВМ в то время на лесозаготовительных предприятиях, эта программа не могла использоваться там для решения различных техникоэкономических задач.

В настоящее время лесозаготовительные предприятия оснащены ПЭВМ, которые применяются, в основном, для решения учетных и бухгалтерских задач, т. е. их возможности используются не в полной мере. В связи с этим алгоритм программы был доработан применительно к возможностям ПЭВМ.

В разработанной на основе данного алгоритма программе расчета показателей движения лесовозных автопоездов исходная информация делится на две группы. Первая вводится при каждом решении задачи и включает, в основном, показатели, характеризующие план и профиль дороги, тип и состояние покрытия, а также наиболее изменяемые параметры автопоезда (нагрузка, масса, коэффициент сцепного веса и т.д.), начальную скорость, номер передачи, с которой начинается движение, и др. Ко второй группе данных относятся показатели, характеризующие конкретный автопоезд. Это - часовой расход топлива на малых оборотах холостого хода; коэффициенты зависимостей, аппроксимирующих тяговую и тормозную характеристики автопоезда для каждой передачи, коэффициенты зависимостей, аппроксимирующих кривую часового хода топлива, и др.

Указанная информация подготовлена для всех основных автопоездов на базе отечественных и зарубежных автомобилей, использующихся для вывозки хлыстов и сортиментов (всего для 21 типа автопоездов).

Алгоритмом программы предусмотрен при единовременном задании исходной информации расчет показателей движения в прямом и обратном направлении (в порожнем и грузовом). В процессе расчетов в конце каждого отдельного участка дороги, а также в точках переключений передач изменения режимов движения выводятся на монитор и печатаются на принтере: скорость и время движения, расход топлива, номер используемых передач, режим движения, скорости ограничения, продольный уклон, радиусы горизонтальных и вертикальных кривых. Кроме вы- водов результатов решения в табличном виде предусмотрен вывод в виде графика на монитор и принтер. Для этого разработана специальная программа.

Использование разработанных выше алгоритма и программы расчета показателей движения лесовозных автопоездов дает возможность получить исходную информацию для обоснования решения широкого круга технико-экономических задач: определение производительности автопоездов, расчеты норм выработки, выбор оптимального типа автопоезда для конкретных условий эксплуатации, прогнозирование производительности и технико-экономических показателей при приобретении новых типов автопоездов, не эксплуатировавшихся ранее в условиях конкретного лесозаготовительного предприятия.

Однако для решения отдельных исследовательских задач потребовалась некоторая модификация и доработка алгоритма программы. Дополнительно разработаны три варианта программы.

1. Алгоритм и программа исследования влияния параметров двигателя и трансмиссии на эксплуатационные и технико-экономические показатели автопоездов.

Алгоритмом предусмотрен ввод данных, аппроксимирующих скоростную и тормозную характеристику двигателя, и нескольких вариантов передаточных чисел трансмиссии (коробки передач, раздаточной коробки и главной передачи). Для всех вариантов трансмиссии определяются зависимости, аппроксимирующие кривые тяговой и тормозной характеристик автомобиля, коэффициенты учета инерции вращающихся масс, допускаемые скорости движения на всех передачах. Последовательно производится расчет показателей движения автопоезда на характерных участках лесовозных дорог и для всех вариантов трансмиссии, что позволит выбрать оптимальный вариант.

2. Версия программы для получения исходной информации, необходимой для оценки и прогнозирования надежности трансмиссии автопоезда и загруженности двигателя.

Для этого определяются и выводятся в табличном виде и в виде диаграмм время и пройденный путь в абсолютных и относительных показателях (процентное отношение) на каждой передаче, время и пройденный путь при полном и частичном использовании мощности, эти же показатели при торможении двигателем, моторным тормозом, в режиме движения накатом, число оборотов коленчатого вала двигателя, расход топлива и др.

Данные показатели определяются для характерных участков лесовозных дорог и дорог общего пользования при различной нагрузке на автопоезд, на основе чего определяются показатели надежности для различных условий эксплуатации. 
3. Версия программы, предназначенная для оценки трассы и профиля дороги по коэффициентам безопасности.

Алгоритмом программы предусмотрен расчет показателей движения по конкретной дороге с учетом ограничений (алгоритм базовой программы) и без учета ограничений по условиям, налагаемым сочетанием геометрических элементов плана и профиля дороги в грузовом и порожнем направлениях, и отношения допускаемых скоростей движения при наличии ограничений скорости и без них (коэффициенты безопасности).

Участки, имеющие коэффициент безопасности 0,81-1, считаются безопасными, 0,6-0,8 - малоопасными, 0,40,6 - опасными и менее $0,4-$ очень опасными.

Опасные для движения автопоездов места на дороге одновременно являются участками с низкими транспортно-эксплуатационными показателями.

Поэтому полученные в результате работы программы графики эксплуатационных скоростей движения, графики максимальных скоростей движения (без учета ограничений) и графики коэффициентов безопасности дают возможность выбрать участки на дороге для первоочередной реконструкции и оценить влияния планируемых мероприятий на эксплуатационные и технико-экономические показатели работы лесовозного транспорта.

Для оценки точности расчета показателей движения, полноты учета факторов, влияющих на режимы и скорость движения лесовозных автопоездов, сходимости графиков, скоростей и режимов лесовозных автопоездов были проведены расчетноэкспериментальные исследования. На участках дороги, для которых имелся исполненный продольный профиль, при движении лесовозных автопоездов фиксировались скорость и время движения, пройденный путь, номера используемых передач КПП, режимы движения: движение при работе двигателя с полным и неполным использованием мощности (внешняя и частичная характеристика), движение накатом, при торможении двигателем, моторным тормозом, колесными тормозами.

Запись показателей движения проводилась с использованием осциллографа и видеоаппаратуры. Для записи на ленту осциллографа пути, пройденного автопоездом, используемых передач, регистрации включения моторного тормоза и колесных тормозов использовались датчики и специально разработанные устройства. Для фиксации времени движения использовался отметчик времени, дающий импульс с частотой 6 Гц.

Скорость движения определялась по формуле:

$v=\frac{s \cdot v_{\Omega}}{l}$, где $l$ - длина протяжки ленты за пройденный путь, мм; $v_{\boldsymbol{r}}$-скорость протяжки ленты, мм/с;

$S$ - путь, пройденный автопоездом за 10 отметок, $s=10 \pi \cdot r_{\partial}$,

$r_{\partial}-$ динамический радиус колеса, м (отмеченный путь сконструирован таким образом, что за один оборот колеса осциллограф фиксирует 2 отметки).

При фиксации показателей движения и обработки полученных материалов с помощью видеоаппаратуры видеокамерой снимались показания спидометра; другие показатели (номер передачи, включение моторного тормоза, торможение двигателем, колесными тормозами, время прохождения контрольных участков пройденного пути) фиксировались из звукового сопровождения. Результаты видеосъемки оцифровывались на компьютере. В результате через каждую секунду определяется скорость движения, затем по формуле

$s=\frac{k \cdot v}{3,6}$

для каждой зафиксированной отметки времени определяется пройденный путь в метрах. В формуле $v$ зафиксированная скорость в км/час; $k$ - тарировочный коэффициент.

С использованием полученных данных строится график скоростей движения пройденного пути, времени движения, номеров используемых передач КПП, использования моторного тормоза, торможения колесными тормозами. Кроме того, на график наносятся уклоны продольного профиля дороги, радиусы вертикальных и горизонтальных кривых, ограничения скорости движения.

Для того чтобы при моделировании движения автопоезда с достаточной точностью учесть сопротивление движению, экспериментально определяется его величина и характер изменения в зависимости от скорости движения.

Для этого выбирались участки дороги длиной 600-800 м с нулевым или близким к нему уклоном. Автопоезд разгонялся до максимальной скорости, затем в начале экспериментального участка включалась нейтральная передача и автопоезд двигался накатом до полной остановки. Диапазон скоростей от 0 до максимальной скорости разбивался на интервалы по 5 км/час. В каждом интервале ускорение условно считалось постоянным и определялось по формуле:

$$
j=\frac{v_{\kappa}-v_{H}}{l},
$$


где $v_{K}$ - скорость в конце участка, м/с; $v_{\boldsymbol{H}}$ - скорость в начале участка, м/с.

Затем рассчитывалось сопротивление движению $\omega$.

$\omega=j-\frac{k \Delta \Omega v^{2}}{G}-i$

Полученные значения обработаны методом наименьших квадратов. Для условий, в которых проводился эксперимент (зимние условия эксплуатации, покрытие - снеголед; летние условия - гравийное покрытие в хорошем состоянии), сопротивление движению определялось зависимостями:

$$
\begin{aligned}
& \omega=0,0155+4,46 \cdot 10^{-5} \cdot v^{2}, \\
& \omega=0,0145+6,812 \cdot 10^{-5} \cdot v^{2} .
\end{aligned}
$$

Для тех же участков, на которых производились экспериментальные исследования по разработанной программе, определялись показатели движения. Для каждого участка строились графики расчетных и зарегистрированных скоростей и режимов движения, которые были совмещены на одном рисунке. Проведенный на первом этапе исследований анализ позволил внести в алгоритм программы некоторые изменения с целью уточнения процесса моделирования движения и повышения сходимости графиков расчетных и фактических (зарегистрированных) скоростей и режимов движения лесовозных автопоездов.

На рис. 1 приведены графики расчетных и фактических скоростей движения автопоездов на базе автомобиля Сису М-162. Анализ графиков расчетных и фактических скоростей и режимов движения показал, что они имеют хорошую сходимость, как по характеру изменения скоростей и режимов движения, так и используемых передач. Подобные расчетноэкспериментальные исследования проведены, кроме того, для автопоездов МА3-509+ТМ3-803 и МА35434+ТМ3-803, всего на 8 участках дорог.

Максимальные отклонения расчетных скоростей движения от фактических не превышают 6\%, тогда как при расчете по наиболее применяемому на лесотранспорте методу равновесных скоростей они доходят до $40 \%$ в целом по дороге, а на отдельных отрезках отличаются вдвое и более.

Графики расчетных (определенных по программе) и фактических скоростей проверены на адекватность по критерию Фишера. Расчеты показали, что модель расчета скорости движения, заложенная в алгоритм программы, адекватна.

Экспериментальная проверка графиков скоростей движения, рассчитанных по методу равновесных скоростей, показала его неадекватность.
Таким образом, проведенные исследования показали, что используемые расчетные зависимости, алгоритм и программа расчета показателей движения лесовозных автопоездов обеспечивают достаточную для решения различных технико-экономических задач точность определения показателей движения лесовозных автопоездов.

Программа использовалась при решении многих технико-экономических задач, возникающих при работе лесовозного автотранспорта. В частности, для обоснования перехода на заготовку леса в сортиментах на одном из предприятий ОАО «Кареллеспром» были определены производительность и техникоэкономические показатели отечественных лесовозных автопоездов (выпускаемых серийно и перспективных), а также нескольких автопоездов на базе зарубежных автомобилей. Проведенные исследования показали, что в условиях предприятия наиболее эффективным является автопоезд ТМ-45-02 + ТМ38966-010 на базе шасси автомобиля КамАЗ-53228, конкурентоспособным является автопоезд на базе МА3-63171. Автопоезда на базе зарубежных лесовозных автомобилей имеют производительность выше на $10-15 \%$, однако эксплуатационные затраты выше в 2,4 раза, а приведенные - более чем в 3 раза.

Для новых типов автопоездов отсутствуют нормы выработки. Путем моделирования движения определена потребность в автопоездах и нормы выработки для перспективных лесовозных автопоездов в условиях конкретного предприятия.

Проведены исследования с целью определения эффективности применения на вывозке автопоездов на базе того же автомобиля КамАЗ-53228, но с двигателем Евро-2 с мощностью 366 л.с. вместо двигателя КамА3-746-13-260. Расчеты показали, что при оснащении автопоезда двигателем в 360 л.с. (Евро-2 КамА3-740-56-360) в составе автопоезда может вместо двухосного применяться трехосный прицеп (например ТМЗ-8966-015). В этом случае грузоподъемность автопоезда повышается с $33,25 \mathrm{~m}^{3}$ до $42,1 \mathrm{~m}^{3}$.

Проведенные исследования по моделированию автопоезда показали, что скорость движения у автопоезда с двигателем 360 л.с. выше, чем у автопоезда с двигателем 260 л.с., несмотря на увеличение массы автопоезда на 9000 кг, а производительность при работе на дорогах лесозаготовительных предприятий выше на $18-24 \%$.

При вывозке леса потребителям эффект от установки на базовом автомобиле двигателя мощностью 360 л.с. и трехосного прицепа выше, причем с увеличением расстояния он возрастает. При расстоянии вывозки 100 км производительность у автопоезда с двигателем 360 л.с. выше, чем в базовом варианте, на 27\% $(60,45$ и 47,6$)$, при расстоянии 150 км - на $30 \%(45,62$ и 35,13$)$, при расстоянии 400 км - на $34,6 \%$; приведенные затраты во всех случаях ниже на $12-14 \%$. 
Программа также применялась для проведения тяговых расчетов, определения производительности автопоездов, выбора оптимального типа автопоезда для конкретных условий эксплуатации, оценки эксплуатационных качеств дорог по коэффициентам безопасности, для обоснования решений при дипломном проектировании.

\section{СПИСОК ЛИТЕРАТУРЫ}

1. Кувалдин Б. И. , Скрыпник В. И. Расчеты на ЭВМ режимов движения лесовозных автопоездов // Известия вузов. «Лесной журнал». 1976. № 6. С. 60-65.

2. Скрыпник В. И., Кочанов А. Н. Новые методы тяговых расчетов на лесовозном транспорте: Учеб. пособие для студентов лесоинженерных специальностей 0901, 0519 высших учебных заведений. Петрозаводск, 1979. С. 76-81.
3. Скрыпник В. И., Кочанов А. Н. Табличный метод расчета показателей неустановившегося движения лесовозных автопоездов // Вопросы механизации и автоматизации работ в лесной промышленности Северо-Запада РСФСР: Межвуз. сб. Петрозаводск, 1977. С. 150-161.

4. Скрыпник В. И., Кочанов А. Н., Пашков Н. В. Номографический метод расчета показателей неустановившегося движения лесовозных автопоездов // Лесосечные, лесоскладские работы и сухопутный транспорт леса: Межвуз. сб. научн. тр. Л., 1977. С. 34-39.

5. Система автоматизированного проектирования продольного профиля. Государственный фонд алгоритмов и программ СССР: Инф. бюллетень / Г. А. Борисов, В. И. Скрыпник, Р. А. Сюкияйнен, Ю. В. Гвоздовский и др.; КарНИИЛП. Петрозаводск, 1977. $68 \mathrm{c}$.

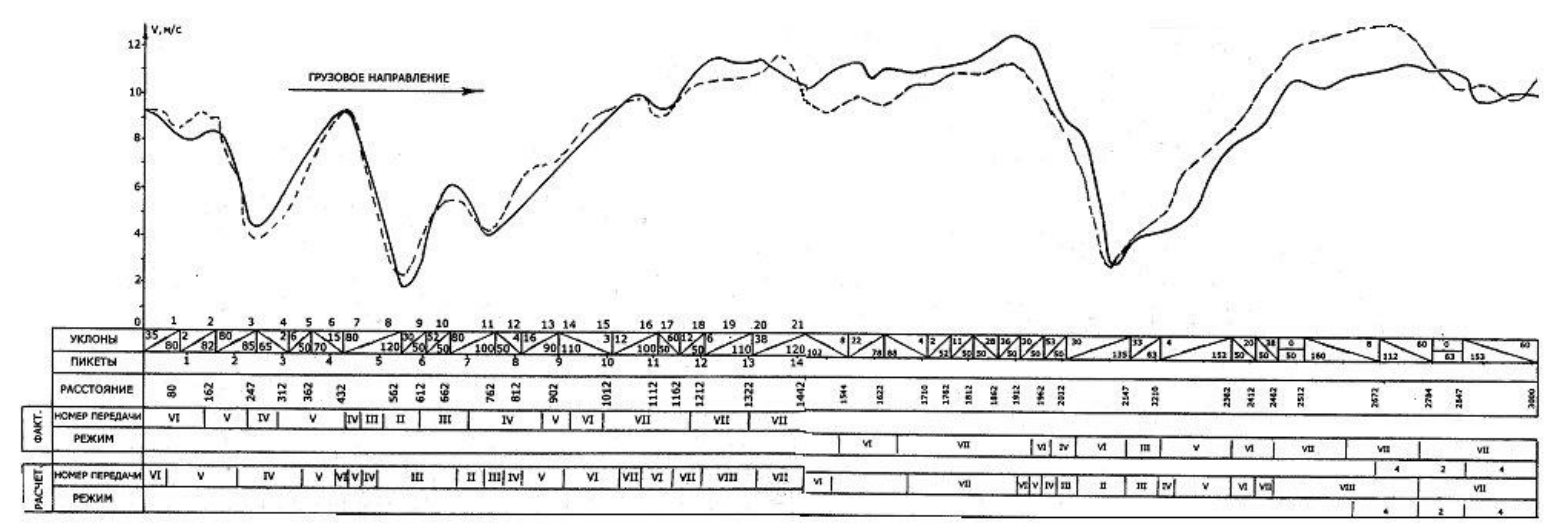

Рис. 1. График скорости движения автопоезда Сису-М-162:

-- фактические скорости движения

